\title{
SUB-MILLIARCSECOND IMAGING OF QUASARS AND ACTIVE GALACTIC NUCLEI. II. ADDITIONAL SOURCES
}

\author{
J. A. ZENSUS \\ Max-Planck-Institut für Radioastronomie, Auf dem Hügel 69, D-53121 Bonn, Germany; and National Radio Astronomy Observatory, \\ 520 Edgemont Road, Charlottesville, VA 22903; azensus@mpifr-bonn.mpg.de \\ E. Ros \\ Max-Planck-Institut für Radioastronomie, Auf dem Hügel 69, D-53121 Bonn, Germany; ros@mpifr-bonn.mpg.de \\ K. I. KeLlermanN \\ National Radio Astronomy Observatory, 520 Edgemont Road, Charlottesville, VA 22903; kkellerm@nrao.edu \\ M. H. COHEN \\ Department of Astronomy, MS 105-24, California Institute of Technology, Pasadena, CA 91125; mhe@astro.caltech.edu \\ R. C. VERMEULEN \\ Netherlands Foundation for Research in Astronomy, Postbus 2, NL-7990 AA Dwingeloo, Netherlands; rvermeulen@nfra.nl
}

AND

M. KADLER

Max-Planck-Institut für Radioastronomie, Auf dem Hügel 69, D-53121 Bonn, Germany; mkadler@mpifr-bonn.mpg.de Received 2002 February 22; accepted 2002 April 30

\begin{abstract}
We report further results from our imaging survey at $15 \mathrm{GHz}(\lambda=2 \mathrm{~cm})$ with the Very Long Baseline Array (VLBA). This paper presents single-epoch images for 39 sources, bringing the total number of objects in the sample to 171. Our sample is representative of a complete unbiased sample, and it will be used for statistical analysis of source properties. We compare the observed brightness temperatures derived from our VLBA observations with those derived from total intensity variations at 22 and $37 \mathrm{GHz}$. These are consistent with intrinsic brightness temperatures in the range $10^{10}-10^{12} \mathrm{~K}$. We also present three new spectroscopic redshift values: $z=0.517 \pm 0.001$ for $0026+346, z=1.591 \pm 0.003$ for $0727-115$, and $z=0.2016 \pm 0.0004$ for $1155+251$.
\end{abstract}

Key words: galaxies: active — galaxies: jets — galaxies: nuclei

\section{INTRODUCTION}

We have been using the Very Long Baseline Array ${ }^{1}$ (VLBA; Napier 1994) since 1994 to study a sample of compact radio sources at $15 \mathrm{GHz}(\lambda=2 \mathrm{~cm})$. We are particularly interested in the scientific aspects related to (1) the morphology of the compact radio sources associated with quasars and radio galaxies (see Zensus 1997), this includes comparisons with results from longer wavelength studies; (2) source variability; (3) statistics of internal kinematics; (4) correlations of the radio results with other properties, e.g., redshift and high-energy luminosity. Our ultimate goal is to derive implications for the relationship between different classes of active galactic nuclei (AGNs), for their physical and cosmological evolution, and for the physics of radio jets.

The images and results of the first 132 sources of our sample were published by Kellermann et al. (1998; hereafter Paper I). The "complete" sample includes 39 additional sources. Images of these objects and three new redshifts are presented and discussed in this paper. ${ }^{2}$ Throughout the paper, we use the Hubble constant $H_{0}=65 \mathrm{~km} \mathrm{~s}^{-1} \mathrm{Mpc}^{-1}$ and the deceleration parameter $q_{0}=0.5$.

\footnotetext{
${ }^{1}$ The National Radio Astronomy Observatory and the Very Long Baseline Array are operated by Associated Universities, Inc., under cooperative agreement with the National Science Foundation.

2 The imaging results of the full project from all epochs are accessible on the Internet at http://www.cv.nrao.edu/2cmsurvey.
}

\section{DEFINITION OF THE SAMPLE}

Our sample consists of 171 sources that were observed during the period 1994-2002. Our initial selection criterion included all sources from the catalog of Stickel, Meisenheimer, \& Kuehr (1994) that are strong $\left(S_{15 \mathrm{GHz}}>1.5 \mathrm{Jy}\right.$ for $\delta>0^{\circ}$ and $S_{15 \mathrm{GHz}}>2 \mathrm{Jy}$ for $0^{\circ}>\delta>-20^{\circ}$ ) and have a flat radio spectrum $\left(\alpha>-0.5, S \sim \nu^{+\alpha}\right)$ at any frequency above $500 \mathrm{MHz}$. The Stickel catalog is complete only at $5 \mathrm{GHz}$, and we have used other measurements or extrapolations to form our $15 \mathrm{GHz}$ sample (see Paper I). We have now observed 93 of the 124 sources that fit the above criteria. The remaining 31 sources $^{3}$ were not observed for a variety of logistic and technical reasons (especially scheduling constraints due to limited observing time), but we consider the 93 sources as representative of an unbiased sample.

An additional 78 sources did not originally fit our criterion but were added because subsequent measurements, including RATAN 600 observations at $15 \mathrm{GHz}$ (Kovalev et al. 1999), showed that they did, although they are not in the Stickel catalog.

\footnotetext{
${ }^{3}$ The missing sources are 0113-118*, 0138-097, 0146+056*, $0248+430^{*}, 0256+075,0332+078,0400+258,0403-102^{*}, 0446+112^{*}$, 0539-057, 0743-006, 0831+557*, 0833+585, 0954+556, 0954+658, $1030+415,1039+811,1147+245,1216+487,1222+131,1418+546$, $1637+574,1725+044,1732+389,1751+441,1936-155^{*}, 2008-159 *$ $2029+121^{*}, 2203-188^{*}, 2216-038,2254+074$. An asterisk indicates that this source was observed in as part of a separate program by Gurvits, Fomalont, \& Kellermann 2002.
} 
Most, if not all, flat-spectrum sources are variable. Thus, at any given time, some sources with mean flux density below the flux limit of a survey will nevertheless be included because they are in a high state, and others with mean flux density above the flux limit will not make it into the sample because they happen to be in a low state. Because of the steep source count or $N(S)$ function, there will be more sources "inappropriately" included than missed. To the extent that the flux density that we used to determine our sample is from a single measurement, the statistical content of the sample is independent of the epochs of measurement; i.e., of the specific sources that make up the sample (see also Drinkwater et al. 1997). In our case, we have a mixture of measurements at $15 \mathrm{GHz}$ and some extrapolations from lower frequencies. Although it is not possible to quantify the statistical effect of this mixture, we conclude that our list is representative of an unbiased, flux-limited sample. As such, it is useful for statistical studies of strong compact radio sources.

Pearson \& Readhead (1988) have presented a complete sample of 65 sources defined by $\delta>35^{\circ},|b|>10^{\circ}$, and $S_{5 \mathrm{GHz}} \geq 1.3 \mathrm{Jy}$. Thirty-two of these do not match either our flux density or our spectral index criterion. We have observed 31 of the remaining 33 objects. Thus, there are only two sources ${ }^{4}$ missing from the complete sample defined by both the selection criteria of the Pearson \& Readhead (1988) sample and ours.

New spectroscopic redshifts for three sources are reported here: $0026+346$ with $z=0.517 \pm 0.001,0727-115$ with $z=1.591 \pm 0.003$, and $1155+251$ with $z=0.2016 \pm$ 0.0004; details are given in Appendix A. This now leaves nine sources out of the 171 without a redshift. Two of these sources are in empty fields (i.e., regions without compact optical features brighter than $20 \mathrm{mag}$ ), and identifications for the others are shown in Table 1. These identifications are taken from the literature with the aid of the NASA/IPAC Extragalactic Database (NED) and the SIMBAD database. The radio galaxies are optical galaxies identified with sources in radio catalogs. Quasars are optically unresolved objects with broad emission lines and, in some cases, absorption lines. BL Lac objects have weak or no emission lines in their spectra.

Figure 1 shows the distribution of redshifts for the sample. The galaxies and BL Lacs are concentrated at low redshift, while the quasars have a broad peak around $z=1$. This is similar to the redshift distribution of the total Stickel et al. (1994) sample; see their Figure 1.

\section{OBSERVATIONS}

Table 1 lists the 39 additional sources and, at the bottom, the 10 sources from Table 1 of Paper I for which new information has become available such as the redshift or identification. We use these new data in the discussion below.

We have continued our observing program described in Paper I with 12 additional epochs, from 1997 August to 2001 March. Throughout, we observed with a bandwidth of $64 \mathrm{MHz}$ using 1 bit samples and left-circular polarization. Each source was observed for 4-6 minutes once per hour over a range of $8 \mathrm{hr}$. Three groups were observed per day,

\footnotetext{
${ }^{4}$ They are $0831+557$ and $0954+556$.
}

with a maximum of 30 sources per day. Typical integration times on each source were 48 minutes.

The amplitude and phase calibration of the visibility data across the frequency channels and across time (usually referred to as a priori calibration and fringe fitting) were carried out for each source within AIPS. Initial hybrid imaging iterations and self-calibration proceeded with a standard DIFMAP script (Pearson et al. 1994); hands-on further processing was carried out whenever obvious problems arose. More details on our data reduction methods are given in Paper I.

\section{DISCUSSION}

Table 2 lists the source properties derived from the contour diagrams that are shown in Figures 2-5. The columns are, successively, the IAU name, the total flux density at 15 $\mathrm{GHz}$ calculated from the VLBA images, the luminosity, the epoch, the lowest contour level shown in Figure 2 (equal to 3 times the rms noise), the major axis, minor axis, and position angle of the restoring beam, the peak and the brightness temperature from the images, and the morphological classification. The equation describing the brightness temperature given in Paper I contains two errors, although the entries in Table 3 (col. [10]) are correct. The correct expression, which we have used in calculating the values of $T_{b}$ in both papers, is $T_{b}=7.6 \times 10^{9} S_{\text {peak }}(1+z) /\left(\theta_{\text {maj }} \theta_{\text {min }}\right)$, where $S_{\text {peak }}$ is the flux density in janskys per beam, and $\theta_{\text {maj }}$ and $\theta_{\min }$ are the major and minor axes of the beam in milliarcseconds (mas).

\subsection{Morphology}

The last column in Table 2 gives the morphological classification, as follows. "C" (compact) refers to sources that are unresolved, or barely resolved. "SS" refers to sources that appear to be single-sided; i.e., the presumed core, identified by compactness, is at one end of the brightness distribution. "Irr" (irregular) refers to those sources where the structure appears two-dimensional. This classification scheme is the same as that used in Paper I, where it is discussed in more detail.

Table 3 summarizes the classification for 169 of the combined set of 171 sources from this paper and from Paper I. Two sources in Paper I could not be classified: 0218+357, which is a lensed object (Patnaik et al. 1993), and 1328+254, which is nearly fully resolved. Objects near zero declination may have low-level artifacts located to the north or south of the real brightness distribution, and in a few cases, the classification is ambiguous ( $\operatorname{see} \S 5$ ).

Most of the sources appear single-sided, which is probably the result of Doppler favoritism: the approaching jet is relativistically boosted and the receding jet is weakened and thus typically not detectable. As expected, all sources from the complete sample that show superluminal internal motions are in this category. Sources that show subluminal motions may have either a single-sided or a double-sided appearance. The internal motions will be discussed in detail by Kellermann et al. (2002).

\subsection{Brightness Temperature}

In many cases, the compact components are unresolved by the $15 \mathrm{GHz}$ VLBA synthesized beam. Most of them may be associated with the central structure, or core. The bright- 
TABLE 1

SOURCE LIST

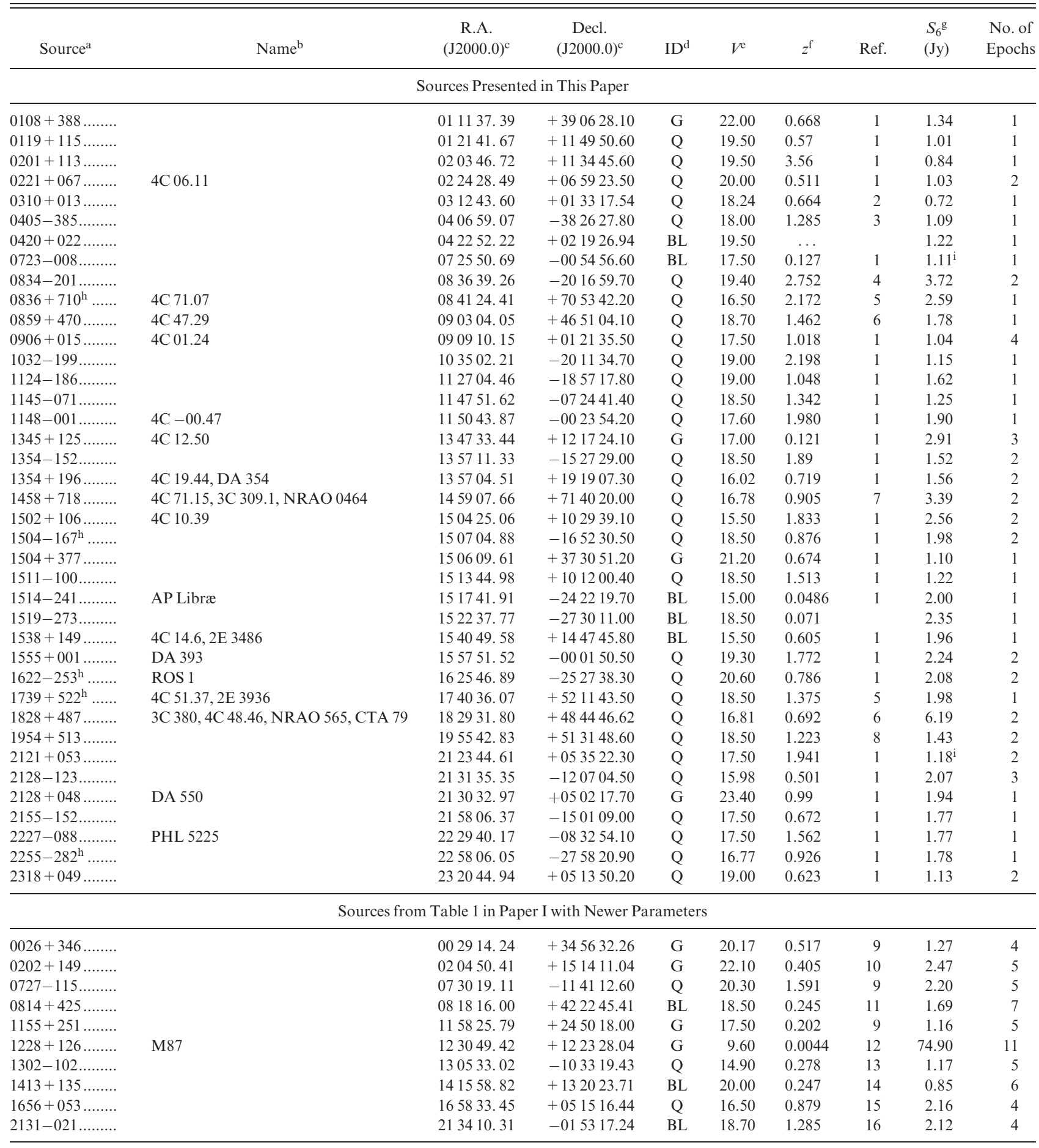

a IAU source designation.

b Alternative source name where appropriate.

c Right ascension and declination (J2000.0). Units of right ascension are hours, minutes, and seconds, and units of declination are degrees, arcminutes, and arcseconds.

d The optical counterpart, denoted as follows: (G) galaxy, (Q) quasar, or (BL) BL Lac object.

e Optical magnitude.

${ }^{\mathrm{f}}$ Redshifts. See reference in next column.

g Flux density at $5 \mathrm{GHz}$, taken mostly from Stickel et al. 1994.

${ }^{\mathrm{h}}$ Detected by the EGRET instrument on the Compton Gamma Ray Observatory (Mattox et al. 1997; Macomb et al. 1999; Hartman et al. 1999).

i Taken from the University of Michigan Radio Astronomy Observatory (UMRAO) Radio Survey Flux Database (http://www.astro.lsa. umich.edu/obs/radiotel/umrao.html; see Hughes, Aller, \& Aller 1992).

ReferenCes.- (1) Kovalev et al. 1999; (2) Hewett, Foltz, \& Chaffee 1995; (3) Stickel et al. 1994; (4) Hewitt \& Burbidge 1989; (5) Hutsemekers 1998; (6) Machalski 1998; (7) Nilsson 1998; (8) Polatidis et al. 1995; (9) see Appendix; (10) Perlman et al. 1998; (11) Lawrence et al. 1996; (12) Smith et al. 2000; (13) Marziani et al. 1996; (14) Wiklind \& Combes 1997; (15) Pravdo \& Marshall 1984; (16) Drinkwater et al. 1997. 


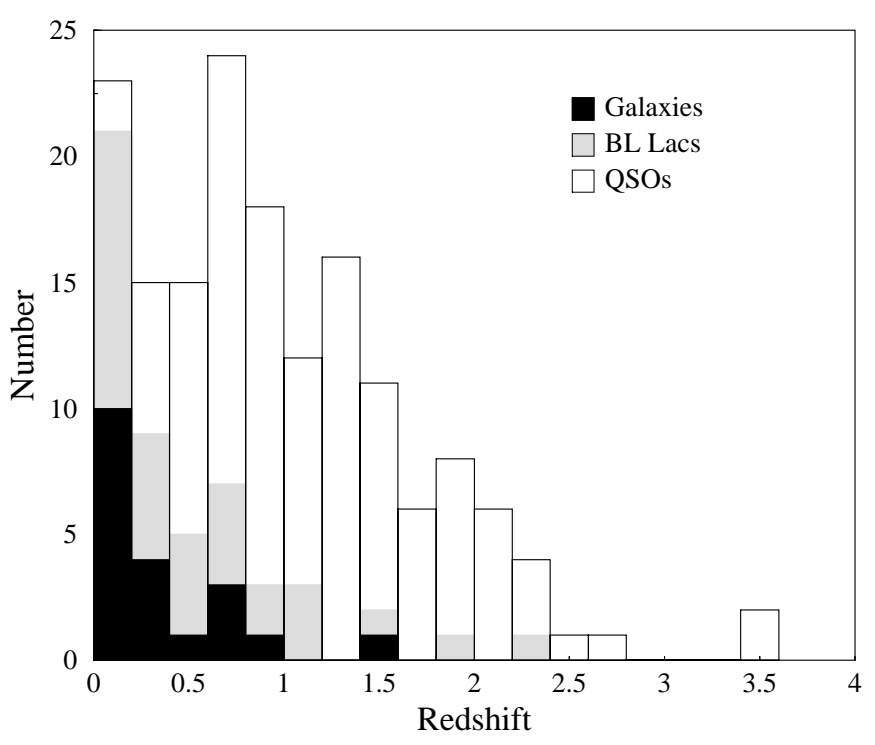

FIG. 1.-Distribution of redshifts for our 171 sources

ness temperature $\left(T_{b}\right)$ in column (10) of Table 2 is calculated using the restoring beam area and, as such, is a lower limit. The existence of much smaller entities with apparently very high values of $T_{b}$ is evidenced by intraday variability (IDV) studies (Kraus et al. 1999; Quirrenbach et al. 2000; Kedziora-Chudczer et al. 2001).

A better estimate of component sizes that are smaller than the restoring beam size can be obtained by fitting elliptical Gaussian models to the visibility data. An example is given in Figure 6, where the visibility for the source $1622-253$ is plotted as a function of $\left(u^{2}+v^{2}\right)^{1 / 2}$. In this case, the visibility is reduced by only $\sim 15 \%$ at 400 million wavelengths; this source can be modeled by two elliptical Gaussian components: one with $2.41 \mathrm{Jy}$, a major axis of 0.14 mas, and an axial ratio of 0.59 (P.A. $=21^{\circ}$ ), giving $T_{b, \mathrm{VLBA}} \sim 3 \times 10^{12}$ $\mathrm{K}$; the other component is circular and has a flux density of $0.08 \mathrm{Jy}$ and a diameter of 0.4 mas at a distance of 0.79 mas $\left(\right.$ P.A. $\left.=-55^{\circ}\right)$ from the compact component.

VLBA data are generally of high quality: the noise is low (typically less than $0.5 \mathrm{mJy}^{-}$beam $\left.^{-1}\right)$, the $(u, v)$-coverage is good, and the relative fringe visibility, after self-calibration, is accurate to a few percent. Therefore, we feel justified in calculating models in this way and using the small sizes to derive brightness temperatures. Table 4 shows $T_{b, \mathrm{VLBA}}$ estimated in this way for sources that show nearly flat visibility plots. These temperatures are calculated in the AGN reference frame. They still must be considered lower limits, since a source like that in Figure 6, which drops slowly to $85 \%$ at the limit of our resolution, can have one or more very small, possibly separated, components.

\subsubsection{Comparison of VLBA and Variability Brightness Temperatures}

Flux density variations provide an alternative approach to estimating $T_{b}$ independently of interferometry measurements. This uses the causality argument that the timescale $\tau$ of a variation cannot be less than the light crossing time of the varying emission region. Taking the two timescales to be equal, $\tau=r / c$, gives the maximal allowed source radius $r$ and hence the minimal allowed brightness temperature, $T_{b, \text { var }}$, from the solid angle $\Omega=\pi r^{2} / D$, where $D$ is the angu- lar size distance to the source. The variability timescale is found from the logarithmic derivative $\tau=d t / d \ln S(t)$ (Burbidge, Jones, \& Odell 1974). In this section, we compare our measured brightness temperatures with those derived by Lähteenmäki \& Valtaoja (1999) from flux density variations at 22 and $37 \mathrm{GHz}$.

Three coordinate frames are involved with these brightness temperatures, and to avoid confusion we use an asterisk for the frame of the relativistically moving plasma; we use no superscript for the frame of the AGN, and we will not define any quantities in the local coordinate frame. The transformations of $T_{b}$, VLBA and $T_{b \text {,var }}$ among these coordinate systems have different powers of $(1+z)$ and $\delta$ (the Doppler factor of the moving plasma). With several assumptions, this allows us to calculate the intrinsic brightness temperature $T_{b \text {,int }}^{*}$. We have two values of $T^{*}$ : $T_{b, \mathrm{VLBA}}^{*}=\delta^{-1} T_{b, \mathrm{VLBA}}$ and $T_{b, \mathrm{var}}^{*}=\delta^{-3} T_{b \text {,var }}$. Strictly speaking, $T_{b, \mathrm{VLBA}}^{*}$ and $T_{b \text {,var }}^{*}$ refer to different plasmas because the measurements are at different wavelengths and at different epochs.

In simple models of relativistic conical jets (e.g., Königl 1981), the radio radiation from the core comes mainly from the region of the jet where the optical depth is unity. This varies with wavelength because the density and magnetic field decrease with distance from the apex. However, the velocity changes slowly, if at all, and hence the Doppler factor can be assumed to be the same for all the frequencies we are using $(15,22$, and $37 \mathrm{GHz})$, which are separated by a factor of only 2.5. The kinetic temperature, which controls the brightness temperature when the optical depth is unity, probably does change slowly along the jet; the biggest effect might be the sideways expansion of the plasma as it moves out. This effect likely is small over a frequency range of 2.5 ; and indeed, the kinetic temperature has been taken as constant in various studies of jets (e.g., Zensus, Cohen, \& Unwin 1995).

The fact that the data for a given source come from different epochs is of concern, but we assume that this is not important. The VLBA measurement refers to the core, which seems to be a permanent feature of these sources, and not to features in the jet, which are evanescent. In addition, the variability timescale appears to change little between outbursts in any one source (Valtaoja et al. 1999).

Thus, we assume that estimates of kinetic temperature derived from the VLBA and from the variability observations should be approximately the same. This is valid only for observations of the flat-spectrum core; it is not valid for the outer steep-spectrum components. Assuming $T_{b, \mathrm{VLBA}}^{*}=$ $T_{b \text {,var }}^{*}$, we derive formulas for estimating the intrinsic temperature $T_{b \text {,int }}^{*}=T_{b}^{*}$ and the Doppler factor:

$$
\begin{gathered}
T_{b, \mathrm{int}}^{*}=\sqrt{T_{b, \mathrm{VLBA}}^{3} / T_{b, \mathrm{var}}}, \\
\delta=\sqrt{T_{b, \mathrm{var}} / T_{b, \mathrm{VLBA}}} .
\end{gathered}
$$

Equation (1) is the same as equation (8) in Lähteenmäki, Valtaoja, \& Wiik (1999).

Table 4 shows $T_{b \text {,var }}$ from Lähteenmäki \& Valtaoja (1999; Table 1) with values converted to $H_{0}=65 \mathrm{~km} \mathrm{~s}^{-1} \mathrm{Mpc}^{-1}$, $q_{0}=0.5$ for those sources that have both a VLBA and a variability brightness temperature. Columns (4) and (5) of Table 4 contain $T_{b \text {.int }}^{*}$ and $\delta$, respectively, calculated from equations (1) and (2). We note first that in every case 
TABLE 2

Source Structure

\begin{tabular}{|c|c|c|c|c|c|c|c|c|c|c|}
\hline $\begin{array}{l}\text { Source } \\
\text { (1) }\end{array}$ & $\begin{array}{c}S_{\text {total }} \\
(\mathrm{Jy}) \\
(2)\end{array}$ & $\begin{array}{c}\text { Luminosity } \\
\left(\mathrm{W} \mathrm{Hz}^{-1} \text { ) }\right. \\
\text { (3) }\end{array}$ & $\begin{array}{l}\text { Epoch } \\
\text { (4) }\end{array}$ & $\begin{array}{c}\text { Contour } \\
(\mathrm{mJy}) \\
(5)\end{array}$ & $\begin{array}{c}\theta_{\text {maj }} \\
(\mathrm{mas}) \\
(6)\end{array}$ & $\begin{array}{c}\theta_{\min } \\
(\mathrm{mas}) \\
(7)\end{array}$ & $\begin{array}{c}\text { P.A. } \\
\text { (deg) } \\
(8)\end{array}$ & $\begin{array}{c}S_{\text {peak }} \\
\left(\text { Jy beam }^{-1}\right) \\
(9)\end{array}$ & $\begin{array}{c}T_{b} \\
(\mathrm{~K}) \\
(10)\end{array}$ & $\begin{array}{c}\text { Structure } \\
\text { (11) }\end{array}$ \\
\hline $0108+388 \ldots \ldots$ & 0.43 & $3.66 \times 10^{26}$ & 1999 Nov 6 & 1.1 & 1.13 & 0.67 & -28.0 & 0.12 & $1.93 \times 10^{9}$ & SS \\
\hline $0119+115 \ldots \ldots$ & 1.37 & $8.92 \times 10^{24}$ & 1998 Oct 30 & 2.2 & 1.09 & 0.51 & -3.9 & 1.11 & $2.36 \times 10^{10}$ & $\mathrm{C}$ \\
\hline $0201+113 \ldots \ldots$ & 0.65 & $8.49 \times 10^{27}$ & 1998 Nov 1 & 0.9 & 1.15 & 0.52 & -7.6 & 0.47 & $2.73 \times 10^{10}$ & SS \\
\hline $0221+067 \ldots \ldots$ & 0.83 & $4.45 \times 10^{26}$ & 1999 Nov 6 & 1.4 & 1.29 & 0.54 & -10.3 & 0.71 & $1.17 \times 10^{10}$ & SS \\
\hline $0310+013 \ldots \ldots$ & 0.16 & $1.38 \times 10^{26}$ & 1998 Nov 1 & 0.8 & 1.29 & 0.55 & -6.2 & 0.11 & $1.91 \times 10^{9}$ & SS \\
\hline $0405-385 \ldots \ldots \ldots$ & 1.01 & $2.68 \times 10^{27}$ & 1998 Nov 1 & 2.4 & 1.43 & 0.44 & 1.4 & 0.79 & $2.20 \times 10^{10}$ & SS \\
\hline $0420+022 \ldots \ldots$ & 1.17 & $\ldots$ & 1999 Nov 6 & 2.7 & 1.37 & 0.54 & -10.6 & 0.91 & $9.31 \times 10^{9}$ & SS \\
\hline $0723-008 \ldots \ldots \ldots$ & 1.01 & $3.90 \times 10^{25}$ & 1997 Aug 18 & 1.8 & 1.03 & 0.47 & 1.1 & 0.60 & $1.06 \times 10^{10}$ & SS \\
\hline $0834-201 \ldots \ldots . .$. & 1.99 & $1.78 \times 10^{28}$ & 1997 Aug 18 & 2.9 & 1.09 & 0.43 & 0.6 & 1.47 & $9.01 \times 10^{10}$ & SS \\
\hline $0836+710 \ldots \ldots$ & 2.24 & $1.39 \times 10^{28}$ & 1998 Mar 19 & 4.5 & 0.80 & 0.52 & -12.3 & 1.49 & $8.58 \times 10^{10}$ & SS \\
\hline $0859+470 \ldots \ldots$ & 0.63 & $2.07 \times 10^{27}$ & 1999 Jul 19 & 1.9 & 1.14 & 0.66 & -7.1 & 0.47 & $1.19 \times 10^{10}$ & SS \\
\hline $0906+015 \ldots \ldots$ & 0.95 & $1.72 \times 10^{27}$ & 1997 Aug 18 & 1.8 & 1.00 & 0.46 & 2.6 & 0.88 & $2.89 \times 10^{10}$ & Irr \\
\hline $1032-199 \ldots \ldots \ldots$ & 1.25 & $7.94 \times 10^{27}$ & 1998 Mar 19 & 7.2 & 1.27 & 0.50 & 0.5 & 0.91 & $3.44 \times 10^{10}$ & SS \\
\hline $1124-186 \ldots \ldots .$. & 2.82 & $5.32 \times 10^{27}$ & 1998 Nov 1 & 2.6 & 1.38 & 0.50 & -4.2 & 2.62 & $5.89 \times 10^{10}$ & $\mathrm{C}$ \\
\hline $1145-071 \ldots \ldots .$. & 0.54 & $1.53 \times 10^{27}$ & 1998 Nov 1 & 1.2 & 1.27 & 0.52 & -2.2 & 0.39 & $1.06 \times 10^{10}$ & SS \\
\hline $1148-001 \ldots \ldots .$. & 0.87 & $4.66 \times 10^{27}$ & 1998 Nov 1 & 0.7 & 1.17 & 0.51 & -1.0 & 0.36 & $1.35 \times 10^{10}$ & SS \\
\hline $1345+125 \ldots \ldots$ & 0.70 & $2.46 \times 10^{25}$ & 1998 Nov 1 & 1.2 & 1.16 & 0.62 & 5.9 & 0.14 & $1.62 \times 10^{9}$ & SS \\
\hline $1354-152 \ldots \ldots \ldots$ & 0.48 & $2.80 \times 10^{26}$ & 1999 Jul 19 & 1.5 & 1.43 & 0.55 & -19.8 & 0.51 & $8.43 \times 10^{9}$ & SS \\
\hline $1354+196 \ldots \ldots$ & 0.75 & $6.28 \times 10^{27}$ & 1997 Aug 18 & 0.9 & 1.14 & 0.46 & -1.5 & 0.43 & $1.84 \times 10^{10}$ & SS \\
\hline $1458+718 \ldots \ldots$ & 1.19 & $1.75 \times 10^{27}$ & 1997 Aug 28 & 1.2 & 0.86 & 0.51 & -13.1 & 0.62 & $2.03 \times 10^{10}$ & SS \\
\hline $1502+106 \ldots \ldots$ & 1.11 & $5.26 \times 10^{27}$ & 2000 Jan 11 & 3.0 & 1.23 & 0.53 & -7.6 & 0.76 & $2.52 \times 10^{10}$ & SS \\
\hline $1504-167 \ldots \ldots \ldots$ & 1.80 & $1.41 \times 10^{27}$ & 1997 Aug 28 & 1.1 & 0.88 & 0.51 & -2.9 & 0.55 & $1.55 \times 10^{10}$ & SS \\
\hline $1504+377 \ldots \ldots$. & 0.73 & $1.14 \times 10^{27}$ & 1997 Aug 18 & 2.4 & 1.16 & 0.46 & 0.0 & 1.18 & $3.13 \times 10^{10}$ & SS \\
\hline $1511-100 \ldots \ldots \ldots$ & 1.34 & $4.70 \times 10^{27}$ & 1997 Aug 18 & 4.5 & 1.20 & 0.50 & 0.1 & 1.13 & $3.61 \times 10^{10}$ & SS \\
\hline $1514-241 \ldots \ldots \ldots$ & 2.23 & $1.32 \times 10^{25}$ & 1997 Aug 18 & 6.0 & 1.16 & 0.44 & -1.3 & 1.21 & $1.91 \times 10^{10}$ & SS \\
\hline $1519-273 \ldots \ldots$. & 1.16 & $1.44 \times 10^{25}$ & 1997 Aug 18 & 1.0 & 1.15 & 0.43 & -2.0 & 1.00 & $1.64 \times 10^{10}$ & SS \\
\hline $1538+149 \ldots \ldots$ & 0.56 & $4.09 \times 10^{26}$ & 1997 Aug 18 & 0.8 & 0.95 & 0.52 & 0.0 & 0.42 & $1.05 \times 10^{10}$ & SS \\
\hline $1555+001 \ldots \ldots$ & 0.71 & $3.21 \times 10^{27}$ & 1997 Aug 18 & 1.1 & 1.08 & 0.50 & 1.6 & 0.56 & $2.19 \times 10^{10}$ & SS \\
\hline $1622-253 \ldots \ldots .$. & 2.52 & $2.91 \times 10^{27}$ & 1997 Aug 18 & 2.3 & 1.26 & 0.46 & -1.7 & 2.34 & $5.51 \times 10^{10}$ & SS \\
\hline $1739+522 \ldots \ldots$ & 1.77 & $5.26 \times 10^{27}$ & 1998 Mar 19 & 3.9 & 0.93 & 0.54 & -1.7 & 1.31 & $4.70 \times 10^{10}$ & $\mathrm{C}$ \\
\hline $1828+487 \ldots \ldots$ & 1.96 & $1.81 \times 10^{27}$ & 1997 Aug 28 & 2.5 & 1.15 & 0.90 & 18.8 & 1.25 & $1.56 \times 10^{10}$ & SS \\
\hline $1954+513 \ldots \ldots$ & 0.94 & $2.30 \times 10^{27}$ & 1998 Mar 19 & 2.0 & 1.02 & 0.55 & 11.6 & 0.51 & $1.54 \times 10^{10}$ & SS \\
\hline $2121+053 \ldots \ldots$ & 2.50 & $1.30 \times 10^{28}$ & 1999 Nov 6 & 4.1 & 1.34 & 0.54 & -9.8 & 2.04 & $6.36 \times 10^{10}$ & SS \\
\hline $2128-123 \ldots \ldots \ldots$ & 1.99 & $1.01 \times 10^{27}$ & 1997 Aug 18 & 2.1 & 1.17 & 0.48 & -0.6 & 1.04 & $2.13 \times 10^{10}$ & SS \\
\hline $2128+048 \ldots \ldots$ & 0.51 & $8.79 \times 10^{26}$ & 1997 Aug 18 & 3.4 & 1.73 & 1.19 & -10.2 & 0.17 & $1.26 \times 10^{9}$ & SS \\
\hline $2155-152 \ldots \ldots \ldots$ & 1.66 & $1.45 \times 10^{27}$ & 1997 Aug 18 & 2.2 & 1.16 & 0.47 & 0.2 & 1.10 & $2.59 \times 10^{10}$ & SS \\
\hline $2227-088 \ldots \ldots \ldots$ & 0.46 & $1.67 \times 10^{27}$ & 1997 Aug 18 & 1.4 & 1.14 & 0.48 & -0.6 & 0.36 & $1.32 \times 10^{10}$ & $\mathrm{C}$ \\
\hline $2255-282 \ldots \ldots \ldots$ & 6.80 & $1.04 \times 10^{28}$ & 1997 Aug 18 & 6.5 & 1.29 & 0.48 & 6.0 & 6.49 & $1.55 \times 10^{11}$ & SS \\
\hline $2318+049 \ldots \ldots$ & 0.75 & $5.70 \times 10^{26}$ & 1997 Aug 18 & 0.9 & 1.06 & 0.51 & -0.7 & 0.69 & $1.57 \times 10^{10}$ & SS \\
\hline
\end{tabular}

a Classification of the structure, as follows: (C) compact, (SS) single-sided, or (Irr) irregular.

$T_{b \text {,var }}>T_{b, \text { VLBA }}$. This is required for $\delta \geq 1$, so in this sense, the data are self-consistent. Values of $\delta$ range from barely relativistic for $0007+016$ to $\delta \sim 50$ for $0235+164$ and $0804+499$. The source $0235+164$ is a known gamma-ray source, and various theories for gamma rays require high values of $\delta$ (Stecker \& Tsuruta 1972; Mattox et al. 1993). Only four sources in Table 4 have EGRET measurements. No general conclusions can be drawn, but the sets of values are consistent with the idea that there is a high Doppler factor in gamma-ray sources.

It perhaps is surprising that the values obtained on the whole correspond well with expectations, since both $T_{b \text {,var }}$ and $T_{b, \mathrm{VLBA}}$ are lower limits, creating an extra margin of uncertainty in the derived values of $T_{b \text {,int }}^{*}$ and $\delta$.

Lähteenmäki et al. (1999) make a similar analysis and show a plot of $T_{b \text {,VLBA }}$ versus $T_{b \text {,var }}$, with diagonal lines indicating values of constant $T_{b \text {.int }}^{*}$. Our results (Table 4) are in agreement with theirs: the points lie between $T_{b \text {.int }}^{*}=10^{10}$ and $10^{12}$.
For many years, a value of $10^{12} \mathrm{~K}$ has been taken as a limit to $T_{b \text {.int }}^{*}$ for a synchrotron radio source in equilibrium because higher temperatures lead quickly to the "inverse Compton catastrophe," which quenches the plasma and reduces its temperature to $10^{12}$ (Kellermann \& Pauliny-Toth 1969). More recently, Readhead (1994) has suggested that the equipartition temperature $T_{\mathrm{eq}} \sim 10^{10}$ or $10^{11}$ is more likely to be appropriate in most objects. Table 4 shows that $T_{b, \text { int }}^{*}=10^{12}$ is approached but not exceeded. The distribution of $T_{b \text {,int }}^{*}$ has a median value of $T_{b \text {,int }}^{*} \approx 10^{11}$, suggesting that they are close to equipartition.

Our ground-based VLBA observations are limited to an angular resolution of about 0.15 mas. For radio sources of a few janskys, this corresponds to a measured brightness temperature of about $10^{12} \mathrm{~K}$, and our determination of $T_{b, \mathrm{VLBA}}$ is only a lower limit. The variability method is limited by time resolution; in the data, we have quoted this is about 1 month, corresponding to a maximum measurable temperature of about $10^{15} \mathrm{~K}$ for $z=1$. Faster flares, corresponding 

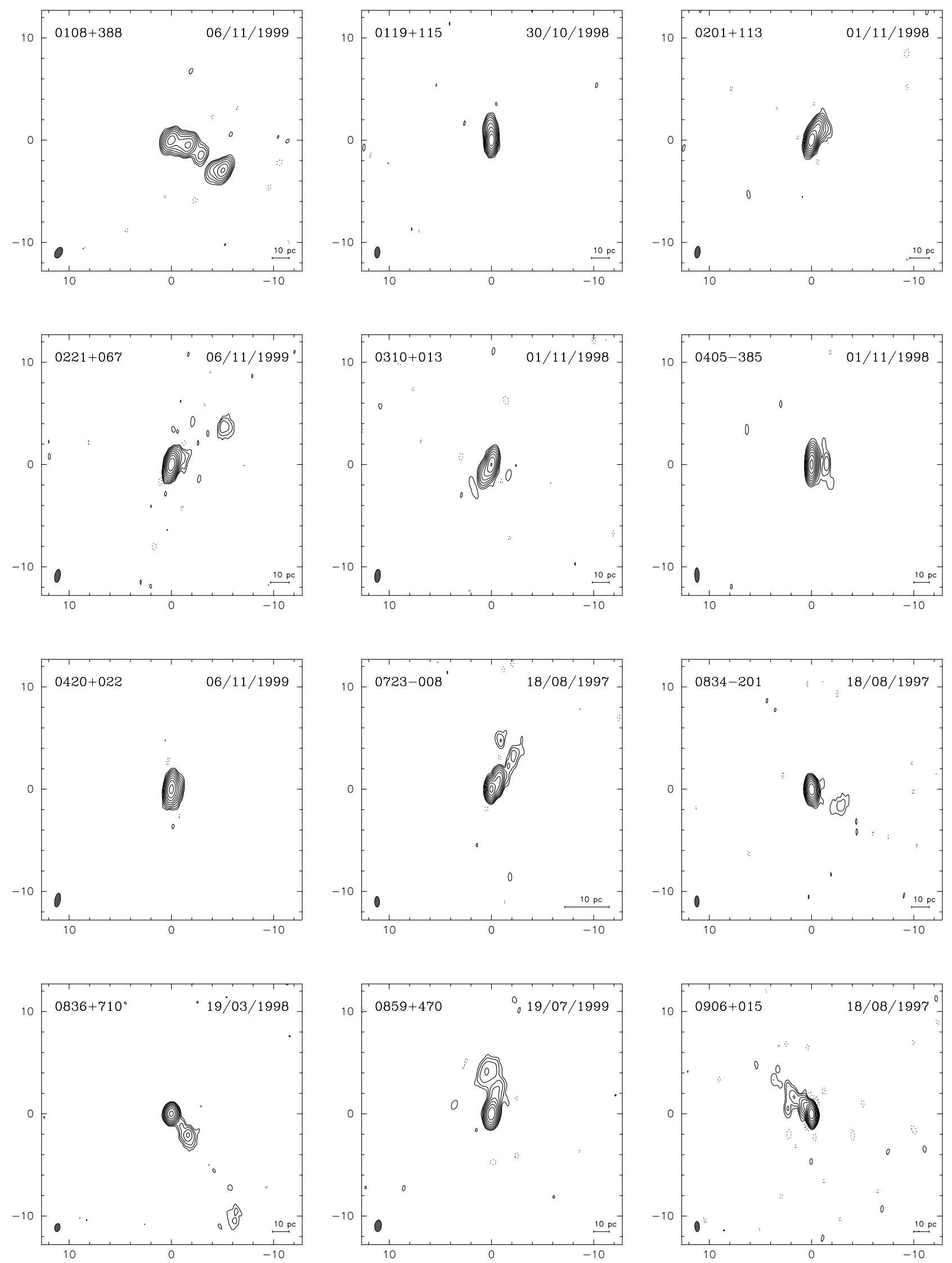

FIG. 2.-Contour maps of the 39 additional sources. The lowest contour level is generally at 3 times the rms noise and is listed in col. (5) of Table 2. The peak flux density in each image is given in col. (9) of Table 2, and the major axis, minor axis, and position angle of the restoring beam are given in cols. (6)-(8). Most images are centered on the brightest component, but for a few of the larger asymmetric sources, we have shifted the center to fit the image in the 25.6 mas box. All of the displayed boxes are 25.6 mas on a side. Each panel also shows a bar representing a linear scale of $10 \mathrm{pc}$. 

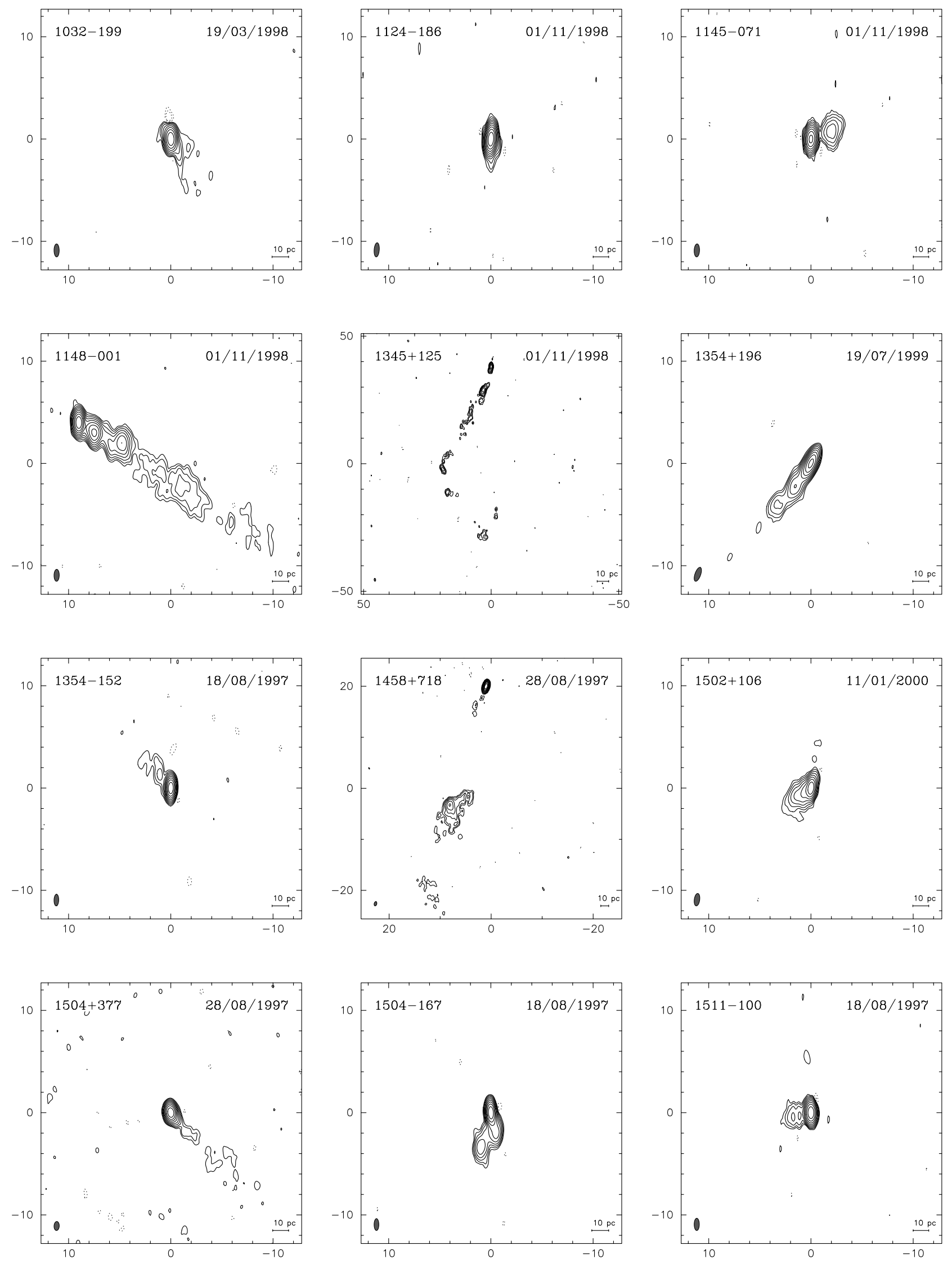

FIG. 3.-Same as Fig. 2, except the displayed box for $1345+125$ is 100.2 mas on a side and for $1458+718,51.2$ mas on a side 

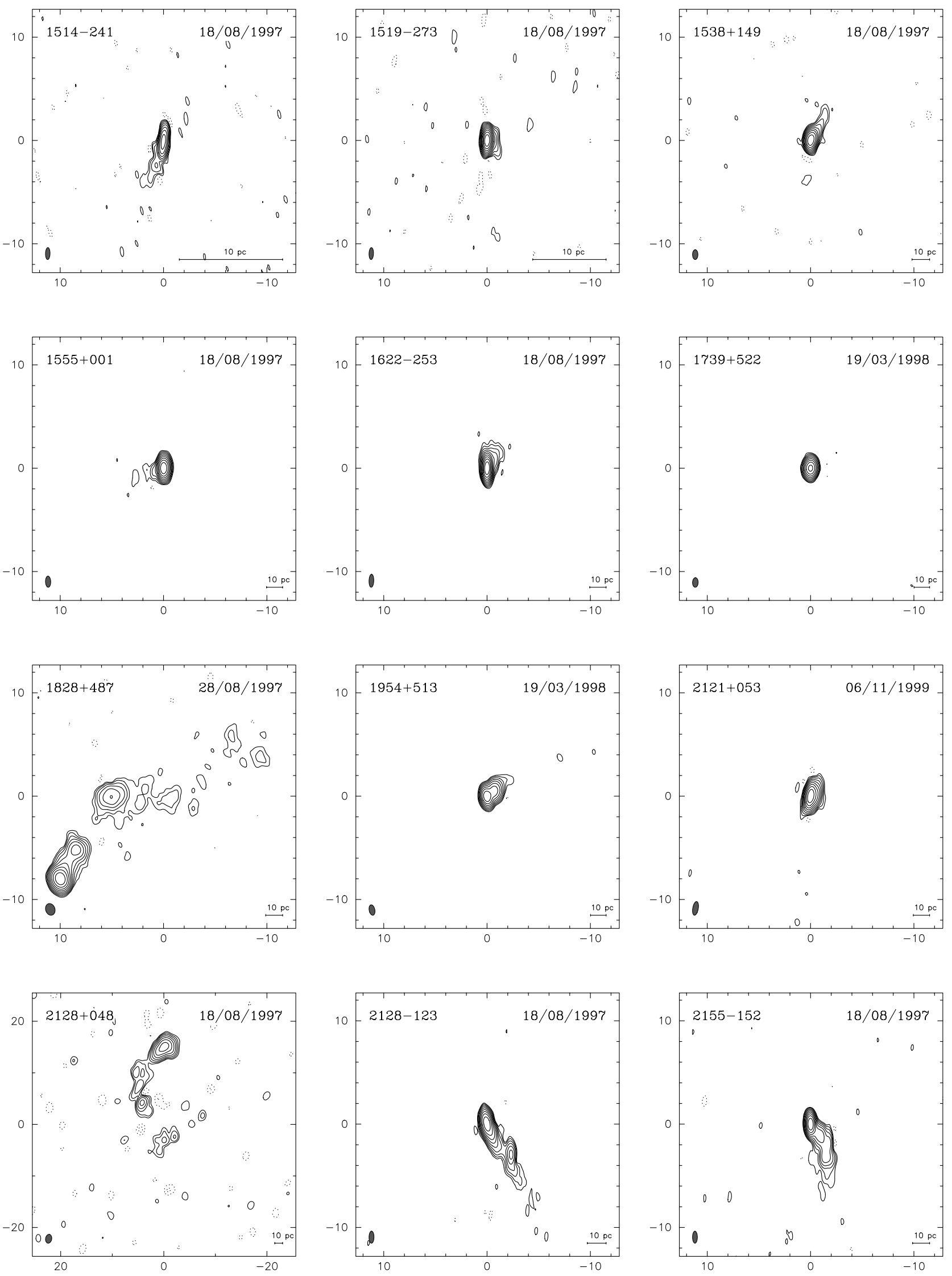

FIG. 4. - Same as Fig. 2, except that the displayed box for $2128+048$ is 51.2 mas on a side 

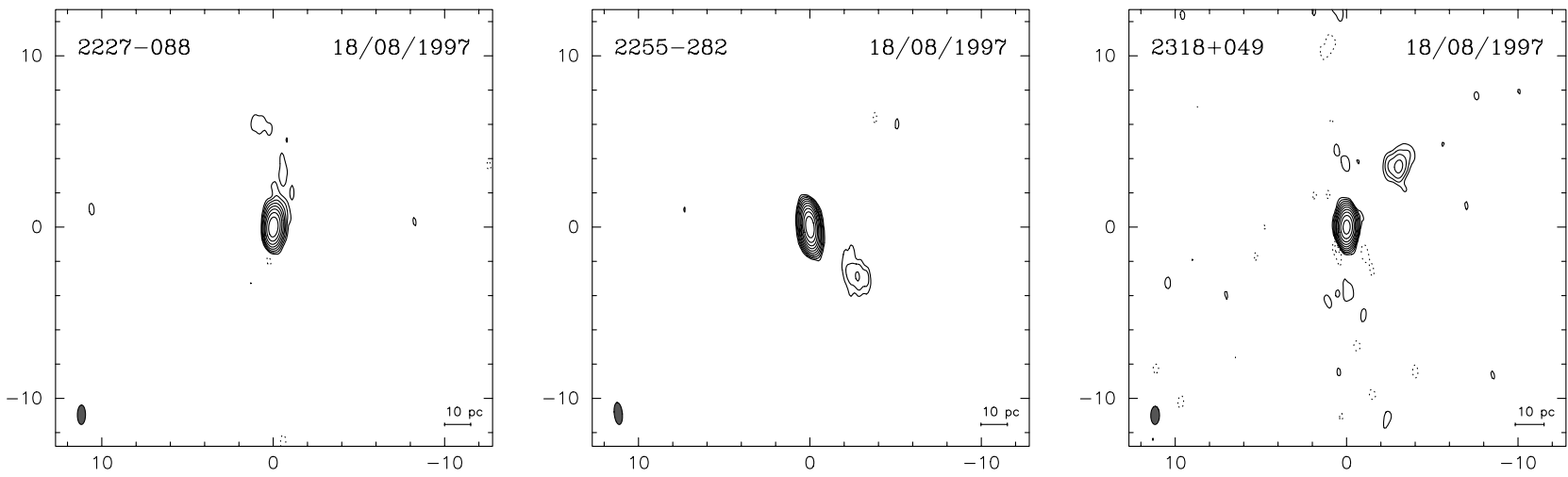

FIG. 5.-Same as Fig. 2

to higher temperatures, are not measurable. These limits are independent, and thus it is particularly interesting that the values in Table 4 are rather close to the values expected on the basis of synchrotron theory and the observations of superluminal motion and X-ray flux. This agreement might mean that the true temperatures are not much above the limits. On the other hand, it may merely reflect a coincidence: 0.1 mas at $z=1$ corresponds to $21 \mathrm{t}-\mathrm{yr}$, which gives an observed light crossing time of about 1 month if the Doppler factor is 20. In this view, the temperatures and Doppler factors in Table 4 are largely given by the observational limits on time and spatial resolution. It is important to test these ideas by improving the limits.

Lähteenmäki \& Valtaoja (1999) actually turn this argument around, by assuming a particular value for $T_{b \text {,int }}^{*}$ to calculate $\delta$. Our procedure is more powerful for the objects in common. However, while we believe we have the requisite high-quality, high-resolution interferometry data, we still rely on the fundamental assumptions that the actual brightness temperatures do not differ grossly from the limits obtained and that $T_{b, \mathrm{var}}^{*} \simeq T_{b, \mathrm{VLBA}}^{*}$.

\section{NOTES ON INDIVIDUAL SOURCES}

In this section, we describe the morphological structure. "RRFID" indicates the observations at 2.3 and $8.4 \mathrm{GHz}$ from the USNO Radio-Optical Reference Frame Image Database (Fey, Clegg, \& Fomalont 1996; Fey \& Charlot 1997, 2000).

0108+388: This source is a compact symmetric object. Proper motions permit an estimated kinematic age of $370 \mathrm{yr}$

TABLE 3

Distribution of Morphology of the Complete Survey

\begin{tabular}{|c|c|c|}
\hline $\begin{array}{l}\text { Structure }^{\mathrm{a}} \\
\text { (1) }\end{array}$ & $\begin{array}{c}\text { Number } \\
\text { (2) }\end{array}$ & $\begin{array}{l}\% \\
(3)\end{array}$ \\
\hline SS ......................... & 142 & 83 \\
\hline $\mathrm{C} \ldots \ldots \ldots \ldots \ldots \ldots \ldots \ldots \ldots \ldots \ldots \ldots \ldots \ldots$ & 13 & 8 \\
\hline DS ........................... & 8 & 5 \\
\hline 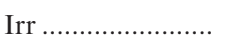 & 6 & 4 \\
\hline
\end{tabular}

a Classification of the structure, as follows: (C) compact, (SS) single-sided, (DS) double-sided, or (Irr) irregular.
(Owsianik, Conway, \& Polatidis 1998). The radio flux density is weakly polarized $(0.30 \pm 0.08 \%$ at $4.8 \mathrm{GHz})$ without significant variations (Aller, Aller, \& Hughes 1992).

0119+115: This source is unresolved by $5 \mathrm{GHz}$ polarization VLBI observations (Gabuzda, Pushkarev, \& Cawthorne 1999). We observed the source at one epoch, and it is slightly elongated to the north. No flux is detected below 2 $\mathrm{mJy}$ beam $^{-1}$ beyond 2 mas from the brightness peak.

$0201+113$ : This system has been extensively studied in the optical due to the peculiar spectroscopic characteristics of a damped Lyman $\alpha$ system toward the host galaxy (Ellison et al. 2001; Kanekar \& Chengalur1997; Oya et al. 1998), H I was detected in the radio (de Bruyn, O'Dea, \& Baum 1996). RRFID shows structure westward at $8.4 \mathrm{GHz}$ and diffuse structure to the southwest at $2.3 \mathrm{GHz}$. In our images, there is an elongated structure to the northwest, not extending beyond 2 mas.

0221+067: This is a flat-spectrum, highly polarized QSO. Our image shows a component 6.3 mas to the northwest of the core. The structure at lower frequencies from RRFID is also elongated in this direction.

0310+013: This compact QSO shows a halo to the west at $4^{\prime \prime}$ in Very Large Array (VLA) images published by Price et

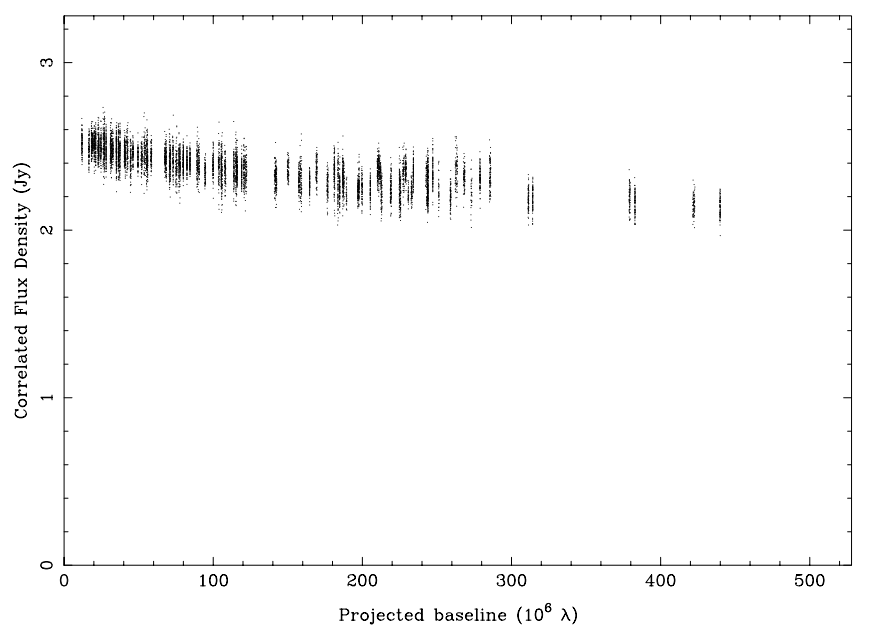

FIG. 6.-Visibility amplitude versus $(u, v)$-radius for the radio source $1622-253$. 
TABLE 4

Brightness Temperatures and Doppler Factors of the Most Compact Sources

\begin{tabular}{|c|c|c|c|c|c|}
\hline $\begin{array}{l}\text { Source } \\
\text { (1) }\end{array}$ & $\begin{array}{c}\log T_{b, \mathrm{VLBA}} \\
\text { (2) }\end{array}$ & $\begin{array}{c}\log T_{b \text {,var }} \\
\text { (3) }\end{array}$ & $\begin{array}{c}\log T_{b, \text { int }}^{*} \\
\text { (4) }\end{array}$ & $\begin{array}{c}\delta \\
(5)\end{array}$ & $\begin{array}{c}\text { EGRET? } \\
\text { (6) }\end{array}$ \\
\hline $0007+016 \ldots \ldots \ldots \ldots$ & 11.26 & 12.21 & 10.79 & 3.0 & \\
\hline $0048-097 \ldots \ldots \ldots \ldots$ & 11.83 & $\ldots$ & $\ldots$ & $\ldots$ & \\
\hline $0235+164 \ldots \ldots \ldots \ldots$ & 11.28 & 14.71 & 9.57 & 51.9 & Yes \\
\hline $0333+321 \ldots \ldots \ldots \ldots$ & 11.68 & 13.50 & 10.77 & 8.1 & No \\
\hline $0607-157 \ldots \ldots \ldots \ldots$ & 12.18 & $\ldots$ & $\ldots$ & $\ldots$ & \\
\hline $0642+449 \ldots \ldots \ldots \ldots$ & 12.04 & 14.75 & 10.69 & 22.6 & \\
\hline $0804+499 \ldots \ldots \ldots \ldots$ & 11.96 & 15.32 & 10.28 & 47.9 & \\
\hline $0808+019 \ldots \ldots \ldots \ldots$ & 11.86 & $\ldots$ & $\ldots$ & $\ldots$ & \\
\hline $0906+015 \ldots \ldots \ldots \ldots$ & 12.48 & $\ldots$ & $\ldots$ & $\ldots$ & \\
\hline $1253-055 \ldots \ldots \ldots \ldots$ & 12.76 & 14.74 & 11.77 & 9.8 & Yes \\
\hline $1308+326 \ldots \ldots \ldots \ldots$ & 11.73 & 14.24 & 10.48 & 18.0 & \\
\hline $1354-152 \ldots \ldots \ldots \ldots$ & 11.73 & $\ldots$ & $\ldots$ & $\ldots$ & \\
\hline $1622-253 \ldots \ldots \ldots \ldots$ & 12.18 & $\ldots$ & $\ldots$ & $\ldots$ & Yes \\
\hline $1638+398 \ldots \ldots \ldots \ldots$ & 11.30 & $\ldots$ & $\ldots$ & $\ldots$ & \\
\hline $1741-038 \ldots \ldots \ldots \ldots$ & 12.08 & 13.92 & 11.16 & 8.3 & \\
\hline $1749+096 \ldots \ldots \ldots \ldots$ & 12.74 & 14.67 & 11.78 & 9.2 & \\
\hline $1758+388 \ldots \ldots \ldots \ldots$ & 11.60 & $\ldots$ & $\ldots$ & $\ldots$ & \\
\hline $1921-293 \ldots \ldots \ldots \ldots$ & 11.89 & $\ldots$ & $\ldots$ & $\ldots$ & \\
\hline $2255-282 \ldots \ldots \ldots \ldots$ & 12.30 & $\ldots$ & $\ldots$ & $\ldots$ & Yes \\
\hline
\end{tabular}

al. (1993). In our $15 \mathrm{GHz}$ image, the source has a compact structure elongated to the southeast.

0405-385: This source is a well-known IDV source (Kedziora-Chudczer et al. 1996, 1997, 1998) with periods of 50\% variability in $1 \mathrm{hr}$. Circular polarization of $-0.101 \%$ was reported by Rayner, Norris, \& Sault (2000). Our 1998 image shows a component to the west at 1.5 mas, compatible with the RRFID images.

0723-008: This BL Lac-type object has a galactic latitude of $+7^{\circ}$. The RRFID images show a jet extending to the northwest at $8.4 \mathrm{GHz}$ up to 7 mas and turning to the north at distances of 40 mas $(2.3 \mathrm{GHz}$ image). This structure is also seen on the 1.6 GHz images of Bondi et al. (1996). Our image from 1997 August shows a clear distinct component at 2 mas to the northwest and more diffuse emission extending to 6 mas from the core.

0834-201: This source is a flat-spectrum blazar (Hewitt \& Burbidge 1989). The first VLBI observations of this radio source were carried out at $1.6 \mathrm{GHz}$ by Kellermann et al. (1971). The VLBA calibrator survey images at $2.3 \mathrm{GHz}$ shows a compact structure extending to the west (Beasley et al. 1996). Our images show a compact source with a very faint component 4 mas away in P.A. of $-100^{\circ}$.

0836+710: This radio source has a highly polarized secondary component at a distance of 1.13 from the core along P.A. $=200^{\circ}$ (Perley 1982). MERLIN-VLBI data show a jet structure extending up to 150 mas in the direction to the outer arcsecond lobe (Hummel et al. 1992). The source has been monitored on parsec scales and shows a complex one-sided core-jet structure along P.A. $=215^{\circ}$ (Krichbaum et al. 1990; Otterbein et al. 1998; Lobanov et al. 1998; Hutchison, Cawthorne, \& Gabuzda 2001; Ros et al. 2001; Lister, Tingay, \& Preston 2001). The jet follows a slightly curved path where components travel outward at

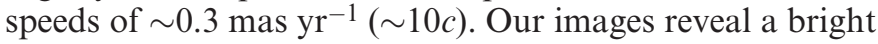
component 2 mas away from the core and a jet extended out to 12 mas. The brightness evolution reported by Peng et al. (2000) shows a slight rise by the mid 1990's reaching a peak around 1997 and decaying afterward. Optical flares and X- ray flares have also been observed (von Linde et al. 1993; Malizia et al. 2000).

0859+470: This source has been observed with VLBI since the 1980's (e.g., Zensus, Porcas, \& Pauliny-Toth 1984; Lawrence et al. 1985; Pearson \& Readhead 1988; Lister et al. 2001, etc.). The RRFID images show an elongated, diffuse structure extending north up to 100 mas at $2.3 \mathrm{GHz}$. A $43 \mathrm{GHz}$ VLBA image by Lister (2001) shows a curved jet structure 1 mas from the core. Our images from 1999 July show two clear components at 2 and 4 mas from the core in P.A. $=10^{\circ}$ and $-10^{\circ}$, respectively.

0906+015: The kiloparsec-scale image from this flat-spectrum QSO published by Murphy, Browne, \& Perley (1993) show a bright component $12^{\prime \prime}$ east of the core. The RRFID images show a jet toward the northeast, reaching 30 mas at $2.3 \mathrm{GHz}$. Our images show jet structure at the same direction, extending out to $3-4$ mas.

1032-199: To our knowledge, no VLBI images from this source have been published before. Our image shows a bright core and some diffuse structure toward the southwest.

1124-186: The images and visibilities from the RRFID and from our data show a compact, unresolved source.

1145-071: Djorgovski et al. (1987) and Kochanek, Falco, \& Muñoz (1999) reported the source as a probable binary quasar, with two optical features at the same redshift and separated by 4.22 in projection. Our image shows a secondary component at the same distance $(2.1$ mas) and position (P.A. $\left.=-63^{\circ}\right)$ as in the RRFID image.

1148-001: Kellermann et al. (1971) reported a compact structure of this source at parsec scales, and interplanetary scintillation observations suggested three compact components at kiloparsec scales (Venugopal et al. 1985). The RRFID images show a jet to the southwest turning to the south beyond 20 mas of the core, extending up to more than 40 mas at $2.3 \mathrm{GHz}$, and with a bright component at $3.2 \mathrm{mas}$, P.A. $=-119^{\circ}$, and a more extended one at 10.9 mas, P.A. $=-123^{\circ}$ at $8.5 \mathrm{GHz}$. Our image from 1998 November reports a conspicuous jet extending to the southwest over 20 
mas, with one component located at 2 mas from the core and the other at 5 mas from the core.

1345+125: The extremely reddened $(U-B=0.73$, $B-I=2.54)$ Seyfert 2 host galaxy contains two nuclei separated by $2^{\prime \prime}\left(\right.$ P.A. $\left.=-75^{\circ}\right)$, suggesting an ongoing merger (Gilmore \& Shaw 1986). Axon et al. (2000) claim that the radio source is associated with the western component of the optical double nucleus from Hubble Space Telescope (HST) observations. This object is similar to Arp 220, the prototype megamaser galaxy. The Arecibo flux density monitoring at 318 and $430 \mathrm{MHz}$ (Salgado et al. 1999) shows no variations over a $14 \mathrm{yr}$ period. The structure of $1345+125$ is discussed in more detail by Lister et al. 2002.

1354+196: This source was observed at $22 \mathrm{GHz}$ by Moellenbrock et al. (1996) in the VSOP Pre-Launch Survey. Our images show a core-jet structure to the southeast, with components at 2 and 5 mas from the core, consistent with RRFID results.

1354-152: Our images show compact structure elongated to the northeast, with diffuse emission out to $\sim 3$ mas.

1458+718: This is a prominent compact steep-spectrum radio source (van Breugel, Miley, \& Heckman 1984; Fanti et al. 1995). Aaron, Wardle, \& Roberts (1997, 1998) studied the polarization and multifrequency properties. Our image from 1997 August reveals a compact core and a more extended component 20 mas to the south, with some extended, diffuse emission. Lower frequency images show emission connecting those two bright features and extending to 50 mas from the core before turning to the east (Aaron et al. 1998).

1502+106: Hardcastle et al. (1997) presented 1.5 and 8.4 $\mathrm{GHz}$ observations of this FR I radio galaxy. Their observations show a broad two-sided jet emanating from a bright, compact core at P.A. $\sim 30^{\circ}$. Martel et al. (1999) show HST images with a subkiloparsec, elongated, and dusty structure centered on its nucleus at P.A. $=163^{\circ}$. The radio jet is orthogonal to the major axis of the dusty structure. The RRFID images display a diffuse parsec structure 5-10 mas to the east of the compact core. Our images show a 3-4 mas structure extending to the southeast.

1504+377: Carilli et al. (1997) reported H I absorption lines toward this inclined disk galaxy. Wiklind \& Combes (1996) found seven different molecular lines toward this object. The 1.6 GHz VLBI images by Polatidis et al. (1995) show an extended jet 100 mas to the southwest. The RRFID images show structure up to 70 mas at $2.3 \mathrm{GHz}$ and 15 mas at $8.4 \mathrm{GHz}$.

1504-167: The $5 \mathrm{GHz}$ VLBI images from this highly polarized quasar (Shen et al. 1997) show a compact structure with three resolved components within 1-2 mas from the core. The RRFID data show a compact structure elongated southward at $8.4 \mathrm{GHz}$. Our images show a nonlinear three-component morphology, with the core, one component at $\sim 2$ mas from the core at P.A. $=-160^{\circ}$, and one at 4 mas from the core at P.A. $=160^{\circ}$.

1514-241: This nearby N-type galaxy displays high variability in the optical, reaching rates of $0.06 \pm 0.01 \mathrm{mag} \mathrm{hr}^{-1}$ (Carini et al. 1991; Bond 1971). The HST images published by Scarpa et al. (2000) show a bright point surrounded by a large, round elliptical host. It has a parsec-scale straight radio jet extending at least $20^{\prime \prime}$ to the east of the core at P.A. $=88^{\circ}$ (Morganti, Killeen, \& Tadhunter 1993). Lister, Marscher, \& Gear (1998) published $43 \mathrm{GHz}$ polarimetric images of this object, showing a jet pointing toward
P.A. $=-171^{\circ}$, within 1 mas of the core, then turning to P.A. $=157^{\circ}$. The RRFID images show a jet toward the southeast. Our image shows structure toward the southeast out to 5 mas.

1519-273: This BL Lac object has a featureless spectrum and a high degree $(5 \%-12 \%)$ of variable optical linear polarization (Impey \& Tapia 1988). It displays IDV at radio frequencies and also in circular polarization (Macquart et al. 2000). It is unresolved at kiloparsec scales (Cassaro et al. 1999). Our image shows a very compact structure with slight extension to the west.

1538+149: This is an optically variable BL Lac object. Ulvestad, Johnston, \& Weiler (1983) report an unresolved structure within $4^{\prime \prime}$ at kiloparsec scales, with hints of extension northward. The RRFID images show emission toward the northwest up to 60 mas at $2.3 \mathrm{GHz}$ and up to 10 mas at $8.4 \mathrm{GHz}$. Our image shows only the inner part of this elongated structure.

1555+001: Our images show a very compact structure with some emission to the east, extending up to 5-6 mas at the $1 \mathrm{mJy}^{\text {beam }}{ }^{-1}$ level.

1622-253: Morganti, Killeen, \& Tadhunter (1993) report an unresolved radio structure at kiloparsec scales. The RRFID images show diffuse emission to the north and northwest without identifiable components from one epoch to another. Our images show a compact structure extending up to 3 mas to the northwest at the $1.5 \mathrm{mJy}^{\text {beam }}{ }^{-1}$ level.

1739+522: A MERLIN image of this highly polarized QSO shows a secondary component $3^{\prime \prime}$ away from the core (Reid et al. 1995). A high-resolution $43 \mathrm{GHz}$ image of the core region by Lister (2001) shows a jet that starts out in an easterly direction and curves over $90^{\circ}$ to the north. Our image shows only an unresolved structure.

1828+487: This is a powerful FR II compact steep-spectrum radio source surrounded by a halo of $14^{\prime \prime} \times 9^{\prime \prime}$ in size (van Breugel et al. 1992). The superluminal motions in this source are described by Polatidis \& Wilkinson (1998). Taylor (1998) reported high rotation measures at parsec scales. The source has a prominent jet extending toward the northwest, which is also visible in our image. Components can be identified at distances of $\sim 4$ and $\sim 10$ mas from the core in P.A. $\sim 30^{\circ}$. Similar structures are visible in the $5 \mathrm{GHz}$ space VLBI image of Lister et al. (2001).

1954+513: The $5 \mathrm{GHz}$ VLA images from Kollgaard, Wardle, \& Roberts (1990) show a north-south double-sided jet, with two components at $5^{\prime \prime}$ and $10^{\prime \prime}$ north of the core and one at $7^{\prime \prime}$ to the south. Our image shows a well-defined jet that extends for approximately 10 mas to the northwest at P.A. $=70^{\circ}$, which agrees with a recent $5 \mathrm{GHz}$ spaceVLBI image by Lister et al. (2001). This jet extends more than 80 mas at $2.3 \mathrm{GHz}$ in the RRFID images.

2121+053: Over the past decades, data from the UMRAO show a slow variation in flux density of this source at 15 $\mathrm{GHz}$ (e.g., Aller et al. 1992). The kiloparsec-scale images from Murphy et al. (1993) show an unresolved source with hints of emission to the east. Our images show a very compact structure, elongated to the west.

2128-123: This is a radio-variable, flat-spectrum QSO. Our images show the same highly curved jet structure as in the RRFID images with a prominent component located 3.7 mas from the core.

2128+048: This very red and weak galaxy has a radio spectrum that is peaked near $600 \mathrm{MHz}$. The $8.4 \mathrm{GHz}$ parsec-scale images from Dallacasa et al. (1998) report a triple- 
component structure with a possible tail at $2.3 \mathrm{GHz}$, compatible with the images from Stanghellini et al. (1997). Our image shows a core-jet structure with an elongated core to the southeast and with further components at 10 mas (P.A. $\left.\sim 160^{\circ}\right)$ and 20 mas (P.A. $\left.=180^{\circ}\right)$ from the core

2155-152: On kiloparsec-scales, this source displays a triple structure extending $66^{\prime \prime}$ in the north-south direction (Perley 1982). The $5 \mathrm{GHz}$ images published by Shen et al. (1998) show a compact core-jet structure aligned with the kiloparsec-scale image. Our image shows a jet toward the south with a bright core and three components up to 7 mas away.

2227-088: Our images show a very compact core with some faint emission extending toward the north up to 6 mas at $1 \mathrm{mJy}^{\text {beam }}{ }^{-1}$, compatible with the RRFID images.

2255-282: This is an optically variable radio source. A gamma-ray flare was detected from EGRET in 1997 (Macomb, Gehrels, \& Shrader 1999). It has been studied at different radio frequencies and scales by Tornikoski et al. (1999) before and after the flare. They present a $5 \mathrm{GHz}$ VLBA image with a jet pointing toward the southwest (also seen in the RRFID images), but their higher frequency observations show only an unresolved source. Our images show a compact core and a component at P.A. $\sim-120^{\circ}$.

2318+049: VLA images (Hutchings et al. 1998) show an unresolved structure slightly elongated along P.A. $=-40^{\circ}$. Our images also show a core-jet structure oriented in the same direction with one distinct component located $\sim 5$ mas off the core (seen also in the RRFID images).

J. A. Z. was supported for this research through a MaxPlanck Research Award. This research has made use of data from the University of Michigan Radio Astronomy Observatory (supported by funds from the University of Michigan); the USNO Radio Reference Frame Image Database (RRFID); the NASA/IPAC Extragalactic Database (NED), which is operated by the Jet Propulsion Laboratory, California Institute of Technology, under contract with the National Aeronautics and Space Administration; and the SIMBAD database, operated at CDS, Strasbourg, France. H. M. Aller, M. C. Aller, J. Armstrong, D. C. Homan, M. L. Lister, A. P. Lobanov, M. Russo, and R. West provided valuable help and important suggestions. R. W. Goodrich and G. B. Taylor collaborated in the optical observations reported in the Appendix.

\section{APPENDIX A}

\section{THREE NEW SPECTROSCOPIC REDSHIFTS}

A 3000 s exposure on $0026+346$ was obtained at the Palomar 200" telescope by R. C. Vermeulen and G. B. Taylor on 1996 January 14, using the COSMIC spectrograph (Kells et al. 1998) with a $300 \mathrm{~g} \mathrm{~mm}^{-1}$ grism and a $1.15 \mathrm{slit}$. Superposed on a red continuum, the emission lines of [O II] $\lambda 3727$ and [O III] $\lambda 4959$ are prominent, as is [O III] $\lambda 5007$, but the centroid of the latter is not well determined, because it falls in the terrestrial atmospheric A-band absorption. From the host galaxy, a Ca II H absorption line, $4000 \AA$ break, and $4300 \AA \mathrm{G}$ band are also clearly visible. All features in the spectrum are consistent with a redshift of $z=0.517 \pm$ 0.001 . In the NED, $0026+346$ is listed with a redshift of $z=0.6$. As far as we have been able to ascertain, this value is a photometric estimate-rather accurate, as we now find - that can be traced back uniquely to a paper by Hutchings et al. (1994).

Observations of $0727-115$ and $1155+251$ were made at the Keck II telescope by M. H. Cohen and R. W. Goodrich on 2000 January 8. The LRIS spectrograph (Oke et al. 1995) was used with the polarimetry module (Goodrich, Cohen, \& Putney 1995) and the $300 \mathrm{~g} \mathrm{~mm}^{-1}$ grating with the $1^{\prime \prime}$ slit. The sky had thin variable clouds.

One exposure of $300 \mathrm{~s}$ duration was obtained for $0727-115$. This object is only $3^{\circ}$ from the galactic plane and is faint but clearly extended. Comparison with HD 245310 ( $V=9.10$; Schmidt, Elston, \& Lupie 1992 ) yields $V \approx 20.3$. C IV $\lambda 1549, \mathrm{C}$ III] $\lambda 1909$, and $\mathrm{Mg}$ II $\lambda 2798$ are prominent and give $z=1.5885,1.5895$, and 1.600 , respectively. The C III] line is cleaner and has a higher signal-to-noise ratio than the others, and the weighted redshift is $1.591 \pm 0.003$.

There apparently is no published spectrum for $1155+251$. The Keck observations show that this bright, narrow-line radio galaxy is reddened and has weak broad $\mathrm{H} \alpha$ emission. The redshift is $0.2016 \pm 0.0004$, based on a direct comparison between the strong lines [O III] $\lambda \lambda 4959,5007$ and the nearby night sky lines [O I] $\lambda 5577$ and $\mathrm{Na}$ I $\lambda 5981$. Other prominent lines include [O II] $\lambda 3727,[\mathrm{Ne}$ III] $\lambda 3869, \mathrm{H} \beta$, $\mathrm{H} \alpha /[\mathrm{N}$ II], and [S II] $\lambda \lambda 6716,6730$.

\section{REFERENCES}

Aaron, S. E., Wardle, J. F. C., \& Roberts, D. H. 1997, Vistas Astron., 41, 225

1998, in ASP Conf. Ser. 144, Radio Emission from Galactic and Extragalactic Compact Sources, ed. J. A. Zensus, G. B. Taylor, \& J. M. Wrobel (IAU Colloq. 164) (San Francisco: ASP), 105

Aller, M. F., Aller, H. D., \& Hughes, P. A. 1992, ApJ, 399, 16

Axon, D. J., Capetti, A., Fanti, R., Morganti, R., Robinson, A., \& Spencer, R. 2000, AJ, 120, 2284

Beasley, A. J., et al. 1996, in IAU Symp. 175, Extragalactic Radio Sources, ed. R. D. Ekers, C. Fanti, \& L. Padrielli (Dordrecht: Kluwer), 527

Bond, H. E. 1971, ApJ, 167, L79

Bondi, M., et al. 1996, A\&A, 308, 415

Burbidge, G. R., Jones, T. W., \& Odell, S. L. 1974, ApJ, 193, 43

Carilli, C. L., Menten, K. M., Reid, M. J., \& Rupen, M. P. 1997, ApJ, 474, L89

Carini, M. T., Miller, H. R., Noble, J. C., \& Sadun, A. C. 1991, AJ, 101, 1196

Cassaro, P., Stanghellini, C., Bondi, M., Dallacasa, D., della Ceca, R., \& Zappalà, R. A. 1999, A\&AS, 139, 601

Dallacasa, D., Bondi, M., Alef, W., \& Mantovani, F. 1998, A\&AS, 129, 219

de Bruyn, A. G., O’Dea, C. P., \& Baum, S. A. 1996, A\&A, 305, 450

Djorgovski, S., Perley, R., Meylan, G., \& McCarthy, P. 1987, ApJ, 321, L17

Drinkwater, M. J., et al. 1997, MNRAS, 284, 85

Ellison, S. L., Pettini, M., Steidel, C. C., \& Shapley, A. E. 2001, ApJ, 549, 770

Fanti, C., Fanti, R., Dallacasa, D., Schilizzi, R. T., Spencer, R. E., \& Stanghellini, C. 1995, A\&A, 302, 317

Fey, A. L., \& Charlot, P. 1997, ApJS, 111, 95 2000, ApJS, 128, 17

Fey, A. L., Clegg, A. W., \& Fomalont, E. B. 1996, ApJS, 105, 299

Fomalont, E. B., Frey, S., Paragi, Z., Gurvits, L. I., Scott, W. K., Taylor, A. R., Edwards, P. G., \& Hirabayashi, H. 2000, ApJS, 131, 95

Gabuzda, D. C., Pushkarev, A. B., \& Cawthorne, T. V. 1999, MNRAS, 307,725

Gilmore, G., \& Shaw, M. A. 1986, Nature, 321, 750

Goodrich, R. W., Cohen, M. H., \& Putney, A. 1995, PASP, 107, 179

Gurvits, L. I., Fomalont, E. G., \& Kellermann, K. I. 2002, in preparation

Hardcastle, M. J., Alexander, P., Pooley, G. G., \& Riley, J. M. 1997, MNRAS, 288, L1

Hartman, R. C., et al. 1999, ApJS, 123, 79

Hewett, P. C., Foltz, C. B., \& Chaffee, F. H. 1995, AJ, 109, 1498

Hewitt, A., \& Burbidge, G. 1989, ApJS, 69, 1

Hughes, P. A., Aller, H. D., \& Aller, M. F. 1992, ApJ, 396, 469

Hummel, C. A., Muxlow, T. W. B., Krichbaum, T. P., Quirrenbach,

A., Schalinski, C. J., Witzel, A., \& Johnston, K. J. 1992, A\&A, 266, 93 
Hutchings, J. B., Dewey, A., Chaytor, D., Ryneveld, S., Gower, A. C., \& Ellingson, E. 1998, PASP, 110, 111

Hutchings, J. B., Neff, S. G., Weadock, J., Roberts, L., Ryneveld, S., \& Gower, A. C. 1994, AJ, 107, 471

Hutchison, J. M., Cawthorne, T. V., \& Gabuzda, D. C. 2001, MNRAS, 321,525

Hutsemekers, D. 1998, A\&A, 332, 410

Impey, C. D., \& Tapia, S. 1988, ApJ, 333, 666

Kanekar, N., \& Chengalur, J. N. 1997, MNRAS, 292, 831

Kedziora-Chudczer, L., Jauncey, D., Tzioumis, A., Reynolds, J., Wieringa, M., Nicholson, G., \& Bignall, H. 1998, IAU Circ., 7066, 2

Kedziora-Chudczer, L., Jauncey, D., Wieringa, M., Reynolds, J., Tzioumis, A. \& Nicholson, G. 1996, IAU Circ., 6418,2

Kedziora-Chudczer, L., Jauncey, D. L., Wieringa, M. H., Walker, M. A., Nicolson, G. D., Reynolds, J. E., \& Tzioumis, A. K. 1997, ApJ, 490, L9

Kedziora-Chudczer, L. L., Jauncey, D. L., Wieringa, M. H., Tzioumis, A.

K. \& Reynolds, J. E. 2001, MNRAS, 325, 1411

Kellermann, K. I., et al. 1971, ApJ, 169, 1

.2002, in preparation

Kellermann, K. I., \& Pauliny-Toth, I. I. K. 1969, ApJ, 155, L71

Kellermann, K. I., Vermeulen, R. C., Zensus, J. A., \& Cohen, M. H. C. 1998, AJ, 115, 1295

Kells, W., Dressler, A., Sivaramakrishnan, A., Carr, D., Koch, E., Epps,

H., Hilyard, D. \& Pardeilhan, G. 1998, PASP, 110, 1487

Kochanek, C. S., Falco, E. E., \& Muñoz, J. A. 1999, ApJ, 510, 590

Kollgaard, R. I., Wardle, J. F. C., \& Roberts, D. H. 1990, AJ, 100, 1057

Königl, A. 1981, ApJ, 243, 700

Kovalev, Y. Y., Nizhelsky, N. A., Kovalev, Y. A., Berlin, A. B., Zhekanis, G. V., Mingaliev, M. G., \& Bogdantsov, A. V. 1999, A\&AS, 139, 545

Kraus, A., Witzel, A., Krichbaum, T. P., Lobanov, A. P., Peng, B., \& Ros, E. 1999, A\&A, 352, L107

Krichbaum, T. P., Hummel, C. A., Quirrenbach, A., Schalinski, C. J., Witzel, A., Johnson, K. J., Muxlow, T. W. B., \& Qian, S. J. 1990, A\&A, 230,271

Lähteenmäki, A.. \& Valtaoja, E. 1999, ApJ, 521, 493

Lähteenmäki, A., Valtaoja, E., \& Wiik, K. 1999, ApJ, 511, 112

Lawrence, C. R., et al. 1985, ApJ, 296, 458

Lawrence, C. R., Zucker, J. R., Readhead, A. C. S., Unwin, S. C., Pearson, T. J., \& Xu, W. 1996, ApJS, 107, 541

Lister, M. L. 2001, ApJ, 562, 208

Lister, M. L., Kellermann, K. I., Vermeulen, R., Zensus, J. A., \& Cohen, M. H. C. 2002, in preparation

Lister, M. L., Marscher, A. P. \& Gear, W. K. 1998, ApJ, 504, 702

Lister, M. L., Tingay, S. J., \& Preston, R. A. 2001, ApJ, 554, 964

Lobanov, A. P., et al. 1998, A\&A, 340, L60

Machalski, J. 1998, A\&AS, 128, 153

Macomb, D. J., Gehrels, N., \& Shrader, C. R. 1999, ApJ, 513, 652

Macquart, J.-P., Kedziora-Chudczer, L., Rayner, D. P., \& Jauncey, D. L. 2000, ApJ, 538, 623

Malizia, A., Bassani, L., Dean, A. J., McCollough, M., Stephen, J. B.,

Zhang, S. N., \& Paciesas, W. S. 2000, ApJ, 531, 642

Martel, A. R., et al. 1999, ApJS, 122, 81

Marziani, P., Sulentic, J. W., Dultzin-Hacyan, D., Calvani, M., \& Moles, M. 1996, ApJS, 104, 37

Mattox, J. R., et al. 1993, ApJ, 410, 609

Mattox, J. R., Schachter, J., Molnar, L., Hartman, R. C., \& Patnaik, A. R. 1997, ApJ, 481, 95

Moellenbrock, G. A., et al. 1996, AJ, 111, 2174

Morganti, R., Killeen, N. E. B., \& Tadhunter, C. N. 1993, MNRAS, 263 1023
Murphy, D. W., Browne, I. W. A., \& Perley, R. A. 1993, MNRAS, 264, 298 Napier, P. J. 1994, in IAU Symp. 158, Very High Angular Resolution Imaging, ed. J. G. Robertson \& W. J. Tango (Dordrecht: Kluwer), 117

Nilsson, K. 1998, A\&AS, 132, 3

Oke, J. B., et al. 1995, PASP, 107, 375

Otterbein, K., Krichbaum, T. P., Kraus, A., Lobanov, A. P., Witzel, A., Wagner, S. J., \& Zensus, J. A. 1998, A\&A, 334, 489

Owsianik, I., Conway, J. E., \& Polatidis, A. G. 1998, A\&A, 336, L37

Oya, S., Iwamuro, F., Tsukamoto, H., \& Maihara, T. 1998, PASJ, 50, 163

Patnaik, A. R., Browne, I. W. A., King, L. J., Muxlow, T. W. B., Walsh, D., \& Wilkinson, P. N. 1993, MNRAS, 261, 435

Pearson, T. J., \& Readhead, A. C. S. 1988, ApJ, 328, 114

Pearson, T. J., Shepherd, M. C., Taylor, G. B., \& Myers, S. T. 1994, BAAS, 26, 1318

Peng, B., Kraus, A., Krichbaum, T. P., \& Witzel, A. 2000, A\&AS, 145, 1

Perley, R. A. 1982, AJ, 87, 859

Perlman, E. S., Padovani, P., Giommi, P., Sambruna, R., Jones, L. R., Tzioumis, A., \& Reynolds, J. 1998, AJ, 115, 1253

Polatidis, A. G., \& Wilkinson, P. N. 1998, MNRAS, 294, 327

Polatidis, A. G., Wilkinson, P. N., Xu, W., Readhead, A. C. S., Pearson, T. J., Taylor, G. B., \& Vermeulen, R. C. 1995, ApJS, 98, 1

Pravdo, S. H., \& Marshall, F. E. 1984, ApJ, 281, 570

Price, R., Gower, A. C., Hutchings, J. B., Talon, S., Duncan, D., \& Ross, G. 1993, ApJS, 86, 365

Quirrenbach, A., et al. 2000, A\&AS, 141, 221

Rayner, D. P., Norris, R. P., \& Sault, R. J. 2000, MNRAS, 319, 484

Readhead, A. C. S. 1994, ApJ, 426, 51

Reid, A., Shone, D. L., Akujor, C. E., Browne, I. W. A., Murphy, D. W., Pedelty, J., Rudnick, L., \& Walsh, D. 1995, A\&AS, 110, 213

Ros, E., Marcaide, J. M., Guirado, J. C., \& Pérez-Torres, M. A. 2001, A\&A, 376, 1090

Salgado, J. F., Altschuler, D. R., Ghosh, T., Dennison, B. K., Mitchell, K. J., \& Payne, H. E. 1999, ApJS, 120, 77

Scarpa, R., Urry, C. M., Falomo, R., Pesce, J. E., \& Treves, A. 2000, ApJ, 532,740

Schmidt, G. D., Elston, R., \& Lupie, O. L. 1992, AJ, 104, 1563

Shen, Z.-Q., et al. 1997, AJ, 114, 1999 1998, AJ, 115, 1357

Smith, R. J., Lucey, J. R., Hudson, M. J., Schlegel, D. J., \& Davies, R. L. 2000, MNRAS, 313,469

Stanghellini, C., O'Dea, C. P., Baum, S. A., Dallacasa, D., Fanti, R., \& Fanti, C. 1997, A\&A, 325, 943

Stecker, F. W., \& Tsuruta, S. 1972, Nature, 235,

Stickel, M., Meisenheimer, K., \& Kuehr, H. 1994, A\&AS, 105, 211

Tornikoski, M., et al. 1999, AJ, 118, 1161

Ulvestad, J. S., Johnston, K. J., \& Weiler, K. W. 1983, ApJ, 266, 18

Valtaoja, E., Lähteenmäki, A., Teräsranta, H., \& Lainela, M. 1999, ApJS, 120,95

van Breugel, W., Miley, G., \& Heckman, T. 1984, AJ, 89, 5

van Breugel, W. J. M., Fanti, C., Fanti, R., Stanghellini, C., Schilizzi, R. T., \& Spencer, R. E. 1992, A\&A, 256, 56

Venugopal, V. R., Ananthakrishnan, S., Swarup, G., Pynzar, A. V., \& Udaltsov, V. A. 1985, MNRAS, 215, 685

von Linde, J., et al. 1993, A\&A, 267, L23

Wiklind, T., \& Combes, F. 1996, A\&A, 315, 86

. 1997, A\&A, 328, 48

Zensus, J. A. 1997, ARA\&A, 35, 607

Zensus, J. A., Cohen, M. H., \& Unwin, S. C. 1995, ApJ, 443, 35

Zensus, J. A., Porcas, R. W., \& Pauliny-Toth, I. I. K. 1984, A\&A, 133, 27 